\title{
The effects of lipids on some aspects of the cellular immune response
}

\author{
BY PIERRE J. GUILLOU \\ Academic Surgical Unit, St Mary's Hospital Medical School, Imperial College of Science, \\ Technology \& Medicine, London W2 1 NY
}

The lipid composition and architecture of the cell membrane play important roles in many aspects of cellular physiology. Structural modifications of the leucocyte membrane might also influence leucocyte function, but until recently this has stimulated only sporadic interest from immunologists. The development of techniques for studying molecular interactions at the lymphocyte cell surface has provided convenient tools for studying the modulatory effects of lymphocyte membrane lipid composition. In parallel with these developments the manufacture of lipid emulsions for administration to patients as part of the energy source in intravenous feeding regimens has, perhaps fortuitously, provided an opportunity to study the effects of such changes in vivo in humans. Consideration of the effects of these lipid emulsions on immunological interactions both in vitro and in vivo may have important clinical implications in addition to the potential value they may have in elucidating the role played by lipids in cell membrane function.

Our own interest in this area has evolved from efforts to administer total parenteral nutrition (TPN) to malnourished tumour-bearing patients in an attempt to augment the depressed anti-tumour lymphocytotoxicity observed in cancer patients. Because of the development of immunotherapy with biological response modifiers in such patients nutritional support could be an important component of this approach. However, some of the themes which we have developed may also have implications for patients with sepsis who also require parenteral nutrition. In the present review, the role of plasma lipoproteins in the modulation of the immune response has not been addressed since this has recently been reviewed elsewhere (Harmony et al. 1986).

\section{LIPID EMULSIONS IN TOTAL PARENTERAL NUTRITION}

The impetus for the provision of intravenous protein and energy substrates in the patient suffering from trauma, cancer or sepsis is the maintenance or restoration of the lean body mass. The N-sparing effect of parenteral nutrition is now well established and amino acids are regarded as an essential part of any intravenous nutritional regimen. As energy sources, glucose and lipid solutions appear to have similar capacities for $\mathrm{N}$ sparing (Nordenstrom et al. 1983). However, it has been recognized that ketone bodies are also a useful source of energy and this has led many to recommend the administration of fat emulsions in TPN on the basis that fatty acids from the hydrolysis of triacylglycerols are the main source of ketones (MacFadyen et al. 1973). Lipid solutions are also an important source of essential fatty acids, particularly those which constitute major components of cell membranes such as linoleic acid and arachidonic acid (Press et al. 1974). These, together with a number of other metabolic advantages (Tweedle, 1978), represent compelling reasons to incorporate lipid emulsions as at least part of the non-protein energy source during TPN. The constituents of the most commonly used 
Table 1. Constituents ( $\mathrm{g} / \mathrm{l})$ of Intralipid (200 mlll) and fatty acid profile of soya-bean oil (mol/100 mol)

\begin{tabular}{llc}
\hline Constituent & Function & $\mathrm{g} / \mathrm{l}$ \\
\hline Soya-bean oil & Fatty acid source & 200 \\
Egg-yolk phospholipid & Emulsifier & 12 \\
Glycerol & To maintain isotonicity & 25 \\
& & mol/100 mol \\
& & 50 \\
Linoleic acid $\left(\mathrm{C}_{18: 2}\right)^{*}$ & & 8 \\
Linolenic acid $\left(\mathrm{C}_{18: 3}\right)^{\dagger}$ & & 26 \\
Oleic acid $\left(\mathrm{C}_{18: 1}\right)$ & 9 \\
Palmitic acid $\left(\mathrm{C}_{16}\right)$ & 3 \\
Stearic acid $\left(\mathrm{C}_{18}\right)$ & 4 \\
Others & & \\
\hline \hline
\end{tabular}

\footnotetext{
* $>$ Arachidonic $\left(\mathrm{C}_{20: 4}\right)$ in vivo.

$\dagger>$ Docosahexaenoic $\left(\mathrm{C}_{22: 6}\right)$ in vivo.
}

solution are summarized in Table 1 from which it can be seen that the principal lipids are of the long-chain fatty acid type.

Two important questions have surrounded the use of lipid emulsions. The first concerns the suggestion that conventional lipid emulsions are immuno-suppressive. The second, and equally controversial, question surrounds the possibility that when employed as an adjunct to cancer therapy, TPN may be deleterious to the medium- and long-term outcome for tumour-bearing patients. Bearing in mind the role of anti-tumour cytotoxic cells in the immuno-surveillance and treatment of malignant disease it is conceivable that these two aspects of nutritional support may be interrelated.

\section{IMMUNOREGULATORY EFFECTS OF LIPID EMULSIONS}

A case report (Freund et al. 1975) on the occurrence of splenic lipidosis in a patient receiving TPN initially raised the possibility that impaired reticulo-endothelial and immunological responses might result from the administration of lipid ernulsions. The essential polyunsaturated fatty acids, linoleic acid $\left(\mathrm{C}_{18: 2}\right)$ and arachidonic acid $\left.\mathrm{C}_{20: 4}\right)$, are prostaglandin precursors and, when ingested, have a depressive effect on murine reticuloendothelial function (Berken \& Benacerraf, 1968). It was subsequently suggested that both the saturated and polyunsaturated fatty acids were capable of inhibiting the response of normal peripheral blood lymphocytes to stimulation with either specific (PPD) or non-specific (PHA) mitogens in vitro in a dose-related manner (Mertin \& Hughes, 1975). In these experiments the polyunsaturated fatty acids appeared to preferentially suppress the proliferation of stimulated rather than unstimulated lymphocytes. Free fatty acids in culture medium are readily incorporated into the cell membrane (Mandel et al. 1978). These substitutions in the cell membrane modify the rate of patching and capping of $\mathrm{H}-2$ surface antigens on murine lymphoma cell lines on exposure to fluoresceinated alloantibodies. This has been interpreted as a reflection of the altered fluidity of the membrane consequent on the modification of its fatty acid profile (Mandel \& Clark, 1978). 
Conversely, Dudrick's group (Ota et al. 1978) claimed that the in vitro co-culture of peripheral blood lymphocytes with relatively high concentrations of lipid emulsions not only did not inhibit lectin and varidase-induced lymphocyte transformation but minimally augmented it. Unfortunately this report lacked adequate controls. Furthermore, the addition of linoleic acid to the cultures in equivalent concentrations to those known to be present in the lipid emulsion was found to almost completely inhibit lymphocyte transformation, an observation which was ignored in the discussion to the paper. Since this initial report, and because of its potential importance with regard to the occurrence of severe sepsis in malnourished patients undergoing TPN (Currie \& Quie, 1971), a number of in vitro and in vivo studies have attempted to address this question. This is of particular relevance to those patients whose nutritional variables are not influenced by preoperative parenteral nutrition and who are known to have a very high incidence of postoperative sepsis. Review of this literature reveals a dichotomy in the evidence which can be summarized according to which type of leucocyte is under study.

\section{Effects of lipid emulsions on monocyte and polymorphonuclear cell functions}

In healthy volunteers, lipid emulsions markedly impair both the random and chemotactin-induced migration of polymorphonuclear leucocytes (Nordenstrom et al. 1979), in vivo and in vitro. In vivo the impaired chemotaxis correlated with the plasma triacylglycerol levels. These findings are compatible with previous in vitro observations that low concentrations of palmitic acid $\left(\mathrm{C}_{16: 0}\right.$; which constitutes approximately $10 \%$ of the fatty acid composition of conventional lipid solution), can selectively inhibit leucocyte chemotaxis (Hawley \& Gordon, 1976). The culture of human polymorphs for brief periods in the presence of varying concentrations of Intralipid (KabiVitrum, Stockholm) equivalent to those obtained during in vivo administration (Cleary \& Pickering, 1983) results in impaired polymorph phagocytic activity. Such cells expressed fewer Fc receptors and exhibited depressed oxidative metabolism. This contrasted with the results obtained when the respiratory studies were performed at the initiation of culture with the Intralipid present, at which time hexose monophosphate shunt activity actually appeared to have been stimulated. Since neither of the other two constituents of Intralipid (egg phospholipid and glycerol) was found to have any effect on these variables, it was concluded that the fatty acid components of the solution initially stimulated the oxidative burst of polymorphonuclear cells resulting in exhaustion of the metabolic pathways involved in respiration, phagocytosis and $\mathrm{Fc}$ receptor availability.

Short-term infusions of Intralipid have also been reported to impair significantly monocyte chemotaxis and phagocytosis in seriously ill postoperative patients as well as healthy volunteers (Fraser et al. 1983). Most authors have also commented on the development of phagoliposomes in the leucocytes studied and it has been suggested that these appearances are associated with functional abnormalities. Both might be prevented by stimulation of the pathway for lipid catabolism via the tissue lipoprotein lipase ( $E C$ 3.1.1.34) system but this has not yet been substantiated (Fraser et al. 1984).

All these investigations seem compatible with the notion that lipid emulsions are at least in part responsible for the high incidence of septic complications seen in malnourished immunocompromised recipients of lipid-based TPN. Despite the presentation of somewhat flimsy evidence to the contrary (Palmblad et al. 1982) these findings are almost entirely in favour of the opinion that the most commonly employed lipid 
emulsions do impair the phagocytic and chemotactic functions of polymorphonuclear leucocytes and monocytes.

\section{Effects of lipid emulsions on lymphocyte function}

In contrast to the effects on polymorph and monocyte function, the position with regard to the influence of lipid emulsions on lymphocyte function is much less clear. There is scant information on the effects of these lipid emulsions on B-lymphocyte function, response to B-cell growth factors or capacity to produce antibody. The majority of in vivo studies of the changes occurring in lymphocytes in the presence of lipid emulsions have not revealed any deleterious effect of this solution on mitogen-induced $T$ lymphocyte transformation, although it may do so in vitro (Ota et al. 1978, 1985; Fraser et al. 1984). Unfortunately most reports have involved short-term ( 2 or $3 \mathrm{~h}$ ) infusions of Intralipid, thus bearing little relationship to its clinical use. We examined T-cells and their function in the context of a prospective randomized controlled clinical study. Our purpose in so doing stemmed from an interest in seeking means of augmenting endogenous immunological responses, particularly those with anti-tumour capacities (Monson et al. 1986).

This study was a $14 \mathrm{~d}$ prospective cross-over trial in which half the patients were administered a lipid-based TPN regimen for the first $7 \mathrm{~d}$ followed immediately by $7 \mathrm{~d}$ of TPN using a wholly carbohydrate-based regimen. The remaining patients received the same regimens over the same time-period but in the reverse order. The nutritional regimens were isoenergetic and isonitrogenous and were prescribed on the basis of calculated metabolic energy expenditure for each individual patient daily.

This study indicated that augmentation of all the immunological variables occurred during the period of lipid-based TPN but not during the wholly carbohydrate-based TPN. Thus, the total numbers of circulating CD3-positive lymphocytes (T-cells) appeared almost to double during the period of lipid-based TPN, apparently owing to a significant increase in the numbers of $\mathrm{CD} 4^{+}$positive $\mathrm{T}$-cells (cytotoxic $\mathrm{T}$-cells). No such changes were observed in the same patients during the periods of entirely carbohydratebased TPN. Production of the entral T-cell lymphokine interleukin-2 (IL-2) was also significantly elevated during TPN with the lipid-based regimen, there being no equivalent changes observed with the carbohydrate-based regimen. At the time we attributed this increased IL-2 production to the increased proportions of circulating numbers of the helper/inducer lymphocyte subset consequent on the presence of the more 'normal' metabolic environment afforded by the lipid-based regimen. We have subsequently re-examined this interpretation but we went on to perform a further study aimed at examining the influence of this lipid-based 'immunorestorative' TPN regimen on lymphocyte-mediated anti-tumour cytotoxicity in cancer patients.

Therefore, we designed a further TPN study with a view to answering two questions (Monson et al. 1988). First, could we reproduce the effects reported in our original study by administering lipid-based TPN for $7 \mathrm{~d}$ to a hompgeneous group of gastrointestinal cancer patients? Second, were the augmented lymphocyte responses previously seen during lipid-based parenteral nutrition followed by identical trends in the generation of anti-tumour cytotoxic responses? Thirty preoperative gastrointestinal cancer patients awaiting surgery gave informed consent to receive $7 \mathrm{~d}$ of TPN with the previously described lipid-based regimen. Lymphocyte function was quantified before initiating 
TPN and the assays were repeated on completion of the $7 \mathrm{~d}$ period of TPN. The assays of lymphocyte function which we selected consisted of measurement of T-lymphocyte transformation in response to a standard mitogen (concanavalin A), IL-2 production in response to this mitogen, natural killer (NK) cell activity, and finally the generation of lymphokine-activated killer (LAK) cell activity following $3 \mathrm{~d}$ of lymphocyte culture in the presence of high dose IL-2. Following retrieval of the operative and pathological findings the patients were subsequently categorized as suffering from localized or advanced disease according to whether or not the disease had spread to involve structures beyond the serosal layer. This was of importance because of our previous finding that it is patients with advanced disease who suffer the most marked perturbations of anti-tumour cytotoxicity (Monson et al. 1987).

As with our first report we confirmed that this lipid-based TPN regimen resulted in the augmentation of IL-2 levels in the supernatant fractions of lectin-activated lymphocytes from these patients at the end of the $7 \mathrm{~d}$ feeding period. However, this appeared to be accompanied by diminished stimulation indices of the lectin-activated lymphocytes and was also in marked contrast to the effects of this regimen on NK activity. The spontaneous lysis of the NK tumour target K562 by peripheral mononuclear cells from these patients was significantly impaired after $7 \mathrm{~d}$ of TPN. Similarly, the killing of K562 by lymphocytes, activated for $3 \mathrm{~d}$ in vitro with high concentrations of IL-2, was also significantly diminished following $7 \mathrm{~d}$ of TPN. When these same activated killer cells were used as effector cells against the DAUDI and COLO 320 cell lines, the same pattern was observed.

A major implication of these findings is that this particular lipid-based TPN regimen should not be used to provide nutritional support for those patients being considered for treatment with biological response modifiers whose therapeutic effects are mediated via the generation of anti-tumour killer cells. This intravenous nutrition regimen is evidently deleterious to the generation of such effectors irrespective of the influence it may have on the phenotypic distribution of lymphocyte subsets and LAK precursors (Ota et al. 1985; Monson et al. 1986). One explanation for this observation is that modification of the lymphocyte membrane saturated:unsaturated fatty acids values may be associated with changes in their lytic potential. It is known that moderate concentrations of oleic acid $\left(C_{18: 1}\right)$ have been found to impair human NK function in vitro (Rice et al. 1981). As will be alluded to later, such changes in the lipid profile of cell membranes result in modification of the fluidity of the cell membrane, with possible effects on lymphocytotoxicity against tumour cells.

Most of the published information relating to the supposed beneficial effects of TPN as an adjunct to surgery or chemotherapy for malignant disease have concentrated on the immediate peritherapeutic period with no regard for the long-term outcome (Copeland et al. 1977; Heatley et al. 1979; Muller et al. 1982). More recently, several clinical studies have suggested that in fact the prognosis may be worse in parenterally-fed patients for reasons unrelated simply to the presence of a central venous feeding catheter. In a study of TPN as an adjunct to the chemotherapy of metastatic colo-rectal cancer the median survival time was significantly less in the intravenously-fed group than in the control group (Nixon et al. 1981). Whether or not lipid emulsions were included in this regimen is unclear. However, they certainly formed part of the protocol described by Shamberger et al. (1982) in a well-designed controlled clinical trial of the use of TPN as an adjunct to surgery and chemotherapy in the treatment of sarcomas. In this study, $71 \%$ of patients in 
the TPN group showed no evidence of disease at the conclusion of therapy compared with $86 \%$ in the control group. More importantly, however, the tumour recurrence rate in the TPN group was $80 \%$ at 1 year with a value of $25 \%$ in the control group, the duration of remission in the TPN group being significantly less than in the non-fed group. Collective reviews of these and other reports on this subject have led a number of authors to question and indeed condemn the use of TPN in the cancer patient, their conclusion being that it is the TPN itself which is responsible for the attenuated prognosis in such patients (Fischer, 1984; Klein et al. 1986). In view of the putative physiological role of NK and LAK cells in the surveillance against micro-metastatic deposits (Hanna \& Fidler, 1980), it is tempting to speculate that the impaired maturation of these cytotoxic killer cells which we have observed following TPN may be one factor contributing to the rather worse prognosis seen in the parenterally-fed patients.

In both our clinical studies (Monson et al. 1986, 1988) we recorded augmented levels of IL-2 in the supernatant fractions of concanavalin A-activated peripheral blood lymphocytes following $7 \mathrm{~d}$ of lipid-based TPN, an effect not seen with the carbohydratebased regimen. The IL-2 bioassay which we used (Gillis et al. 1978) measures free unbound IL-2 in the lymphocyte supernatant fraction at the time of the assay. The free IL-2 content of a given supernatant fraction must represent the residual balance between the IL-2 being elaborated and released by the activated T-cells and that which is being bound by pre-existing receptors or newly generated receptors on the surface of the activated T-cells. Increased levels of IL- 2 in the supernatant fraction, therefore, may reflect not only increased production of IL-2 but also diminished binding of IL-2 to the high-affinity receptor. Accordingly, an alternative interpretation of our findings is that there is reduced binding of IL-2 to its receptor, rather than increased production. This might have occurred as a consequence of some change either in the expression or function of the IL-2 receptor during the lipid-based TPN. Such a hypothesis would also explain the reduced NK activity observed in vivo during the lipid-based TPN in our second study because of the physiological role which IL-2 and other cytokines play in the maintenance of NK activity. The reduced capacity to stimulate LAK activity after lipid-based TPN compared with that before TPN might also be explained on the same basis.

Because of the known effects of different unsaturated fatty acids on leucocyte membranes, in addition to the fact that lipid emulsions adversely affect polymorph and monocyte function, it seems reasonable to suggest that the lipid emulsion is the component of the TPN regimen which is responsible for these modifications of lymphocyte function. The provision of at least some lipid is considered necessary to prevent essential fatty acid deficiency and provide non-protein energy in most TPN regimens. We felt, therefore, that it was important to examine whether or not our hypothesis on the interference by lipid emulsions with IL-2 binding or IL-2 receptor function was tenable on the basis of in vitro studies using defined reagents. In addition, newer lipid formulations which reputedly provide the same level of energy but contain lower concentrations of long-chain fatty acids are now available for clinical use (Sedman et al. 1990). We investigated whether or not these newer formulations also possessed immunomodulatory properties.

These immunological effects were studied in vitro in the presence of several commercially available lipid emulsions including the standard Intralipid, a similar egg phospholipid-soya-bean emulsion, Lipofundin S (Braun, Melsungen, Germany) and a 
more recently developed emulsion in which only $50 \%$ of the energy is supplied as long-chain triacylglycerols (LCT), the remainder being present as medium-chain $\left(\mathrm{C}_{6}-\mathrm{C}_{10}\right)$ triacylglycerols (MCT; Braun). In vitro, all three emulsions inhibited lymphocyte proliferation and LAK cell induction. However, these emulsions exerted differential effects, the standard emulsions consistently producing greater levels of inhibition of the lymphocyte responses than the MCT (Sedman et al. 1990).

The proliferation (as assessed by cell number and $\left[{ }^{3} \mathrm{H}\right]$ thymidine uptake) of growth factor-independent lymphoblastoid cell lines such as K562, DAUDI and RAJI, was not influenced by these emulsions, excluding a toxic effect. In contrast, the addition of the LCT emulsions to cultures of the IL-2-dependent murine T-cell line CTLL-2 impaired the proliferation of this clone even at low concentrations. A similar trend was seen with the MCT but was much less marked. Identical effects on human lymphocyte proliferation were observed when these emulsions were added to cultures of resting lymphocytes incubated for $3 \mathrm{~d}$ in the presence of IL-2, the MCT again having significantly less suppressive influence than the soya-bean emulsions. However, the in vitro findings with MCT have been confirmed in a further clinical trial in which the MCT was found to be less suppressive of NK-cell activation with IL-2 (measured as LAK cytotoxicity) than a regimen containing either Intralipid or no lipid as the energy source (Sedman et al. 1991).

These in vivo and in vitro findings suggest that these emulsions do interfere with IL-2 receptor interactions and we are currently examining whether or not the binding or release of other lymphokines such as interferon gamma are impaired in response to mitogenic signals such as IL-2 and the anti-CD3 monoclonal antibody. If so, then this might indicate a general effect of these emulsions on lymphocyte receptor-ligand interactions. This would also have implications for the septic patient who requires TPN, since the early events in T-lymphocyte activation are common to other receptormediated activation systems such as activation of B-cells by B-cell differentiation factor or growth factor (BCDF or BCGF).

Why do these long-chain fatty acids have these interesting and potentially deleterious effects? It is considered that the lipid constitutents of the cell membrane are in a fluid and homogeneous phase but free fatty acids can readily enter the cell membrane and influence the fluidity of the lipid bilayer (Stubbs et al. 1980). cis-Unsaturated fatty acids particularly can disorder the membrane interior and render the cell membrane more rigid (Klausner et al. 1980). T-lymphocytes are particularly susceptible to long-chain fatty acid insertions because, unlike other lymphocytes, they do not possess the $\delta$-desaturase enzyme (Tebbey \& Buttke, 1990). The function of certain cell surface receptors is dependent on their lateral mobility within the cell membrane. Consequently, any change in cell membrane fluidity may modify the lateral movement of these receptors or their components. Interestingly, this phenomenon is seen only with unsaturated fatty acids and not following the incorporation of saturated fatty acids into the cell membrane. Clearly then, alteration of membrane fluidity by modifying the lipid composition of lymphocyte cell membranes with unsaturated fatty acids (present in high concentrations in the lipid emulsions used for TPN) can markedly influence receptor-ligand interactions.

The high affinity IL-2 receptor is a two-chain ( $\alpha$ and $\beta$ ) structure possessing external, transmembrane and intracellular domains. Either the $\alpha$ or the $\beta$ chain can separately bind IL- 2 but cannot alone initiate the transmembrane signal. Thus, the $\alpha$ chain and/or the $\beta$ chain may both represent the low-affinity receptor. When IL-2 is bound (at 
separate sites) by both the $\alpha$ and the $\beta$ chains simultaneously the high-affinity receptor is formed and, in the presence of bound IL-2, endocytosis of the receptor-ligand complex occurs and the signal for intracellular T-cell activation is initiated.

The apposition of the $\alpha$ and $\beta$ chains depends on their lateral mobility in the cell membrane and any change of the latter would presumably interfere with the aforementioned sequence of events. It is possible, therefore, that the incorporation of unsaturated fatty acids from the lipid emulsions, where they are present in relatively high concentrations, might provide an explanation for the interference with IL-2-mediated reactions in the clinical and experimental studies described previously. Thus, in the two clinical studies, elevated levels of IL-2 in supernatant fractions from lectin-activated lymphocytes following lipid-based TPN might be explained by failure of apposition of the two chains of the IL-2 receptor. If lectin activation stimulates IL-2 production independently of initial receptor binding, then this failure to co-bind IL-2 would ultimately lead to a diminished generation of new IL-2 receptor components and result in increased levels of IL-2 in the supernatant fractions. Similarly, the failure of IL-2 to activate NK and LAK cells in the second clinical study might also be explained on the same basis. Finally, this hypothesis is an attractive explanation for the in vitro studies described previously, the advantage conferred by the MCT solutions simply resulting from the fact that these solutions contain fewer of the long-chain unsaturated fatty acids.

Unfortunately we have recently accrued information which is difficult to rationalize with this hypothesis. The intracellular signal induced by the binding of IL-2 to its receptor is mediated via the activation of tyrosine phosphokinases upstream of the pathway which results in inositol triphosphate formation. Using a western blot technique we have followed the activation of intracellular tyrosine phosphokinase in the immediate period following IL-2-receptor binding. These preliminary findings reveal that in the presence of lipid emulsions the appearance of activated tyrosine phosphokinase occurs much earlier than it does in unmodified cells (J. F. Dye and P. J. Guillou, unpublished results). This would be compatible with increased membrane fluidity in the presence of LCT and, thus, the generation of an early signal. In fact, we have found this to be accompanied by sustained cell membrane depolarization. How this relates to the subsequent functional defect is currently the focus of considerable attention in our laboratory.

Equally, these tools are vital to dissect the nature of the defects which lipid emulsions induce in polymorphs and monocyte/macrophage function. The response to sepsis is mediated by these cells, bacterial lipopolysaccharide inducing the expression of the proinflammatory cytokines IL-1, IL-6 and tumour necrosis factor $\alpha$. The effects of lipids on these components of the sepsis response may provide insights which permit the logical administration of appropriate lipids to the septic patient who so often requires parenteral nutrition.

\section{REFERENCES}

Berken, A. \& Benacerraf, B. (1968). Depression of reticulo endothelial function by ingested lipids. Proceedings of the Society of Experimental Biology and Medicine 129, 793-801.

Cleary, T. G. \& Pickering, L. K. (1983). Mechanisms of intralipid effect on polymorphonuclear leukocytes. Journal of Clinical and Laboratory Immunology 11, 21-26.

Copeland, E. M., Daley, J. M. \& Dudrick, S. J. (1977). Nutrition as an adjunct to cancer treatment in the adult. Cancer Research 37, 2451-2459. 
Currie, C. R. \& Quie, P. J. (1971). Fungal septicaemia in patients receiving parenteral hyperalimentation. New England Journal of Medicine 285, 122-125.

Fischer, J. E. (1984). Adjuvant parenteral nutrition in the patient with cancer. Surgery 96, 578-581.

Fraser, I., Neoptolemos, J., Darby, H. \& Bell, P. R. F. (1984). The effects of intralipid and heparin on human monocyte and lymphocyte function. Journal of Parenteral and Enteral Nutrition 8, 381-384.

Fraser, I., Neoptolemos, J., Woods, P., Bowry, V. \& Bell, P. R. F. (1983). The effect of Intralipid on human lymphocyte and monocyte function. Clinical Nutrition 2, 37-40.

Freund, U., Krausz, Y., Levij, I. S. \& Eliakim, M. (1975). Iatrogenic lipidosis following prolonged intravenous hyperalimentation. American Journal of Clinical Nutrition 28, 1156-1158.

Gillis, S., Ferus, M. M., Ou, W. \& Smith, K. A. (1978). T-cell growth factor: parameters of production and a quantitative microassay for activity. Journal of Immunology 120, 2027-2031.

Hanna, N. \& Fidler, I. J. (1980), Role of natural killer cells in destruction of circulating tumour emboli. Journal of National Cancer Institute 65, 801-805.

Harmony, J. A. K., Akeson, A. L., McCarthy, B. M., Morris, A. R., Scupham, D. W. \& Grupp, S. A. (1986). Immunoregulation by plasma lipoproteins. In Biochemistry and Biology of Plasma Lipoproteins. [A. M. Scanu and A. A. Spector, editors]. New York: Marcell Dekker Inc.

Hawley, P. \& Gordon, G. B. (1976). The effects of long-chain fatty acids on human neutrophil function and structure. Laboratory Investigation 34, 216-223.

Heatley, R. V., Williams, R. H. P. \& Lewis, M. H. (1979). Preoperative intravenous feeding - a controlled clinical trial. Postgraduate Medical Journal 55, 541-545.

Klausner, R. D., Kleinfeld, A. M., Hoover, R. L. \& Karusvsky, M. J. (1980). Lipid domains in membranes. Evidence derived from structural perturbation induced by free fatty acids and lifetime heterogeneity analysis. Journal of Biological Chemistry 255, 1286-1295.

Klein, S., Simes, J. \& Blackburn, G. L. (1986). Total parenteral nutrition and cancer clinical trials. Cancer 58, 1378-1386.

MacFadyen, B. V., Dudrick, S. J., Tagudar, E. P., Maynard, A. T., Law, D. K. \& Rhoads, J. E. (1973). Triglyceride and free fatty acid clearances in patients receiving complete parenteral nutrition using a ten per cent soybean oil emulsion. Surgery, Gynaecology and Obstetrics 137, 813-820.

Mandel, G. \& Clark, W. (1978). Functional properties of EL-4 tumor cells with lipid altered membranes. Journal of Immunology 120, 1637-1642.

Mandel, G., Shimizu, S., Gill, R. \& Clark, W. (1978). Alteration of the fatty acid composition of membrane phospholipids in mouse lymphoid cells. Journal of Immunology 120, 1631-1636.

Mertin, J. \& Hughes, D. (1975). Specific inhibitory action of polyunsaturated fatty acids on lymphocyte transformation induced by PHA and PPD. International Archives of Allergy and Applied Immunology 48, 203-210.

Monson, J. R. T., Ramsden, C. W., Giles, G. R., Brennan, T. G. \& Guillou, P. J. (1987). Lymphokineactivated killer (LAK) cells in patients with gastrointestinal cancer. Gut 28, 1420-1425.

Monson, J. R. T., Ramsden, C. W., MacFie, J., Brennan, T. G. \& Guillou, P. J. (1986). Immunorestorative effect of lipid emulsions during total parenteral nutrition. British Journal of Surgery 73, 843-846.

Monson, J. R. T., Sedman, P. C., Ramsden, C. W., Brennan, T. G. \& Guillou, P. J. (1988). Total parenteral nutrition adversely influences tumour-directed cellular cytotoxic responses in patients with gastrointestinal cancer. European Journal of Surgery and Oncology 14, 435-443.

Muller, J. M., Brenner, U., Dienst, C. \& Pichlmaier, H. (1982). Preoperative parenteral feeding in patients with gastrointestinal carcinoma. Lancet $\mathbf{i}, 68-71$.

Nixon, D. W., Moffit, S. \& Lawson, D. H. (1981). Total parenteral nutrition as an adjunct to chemotherapy of metastatic colorectal cancer, Cancer Treatment Reports 65, 121-126.

Nordenstrom, J., Askanazi, J. \& Elwyn, D. H. (1983). Nitrogen balance during total parenteral nutrition. Annals of Surgery 197, 27-33.

Nordenstrom, J., Jarstrand, C. \& Wiernick, A. (1979). Decreased chemotactic and random migration of leukocytes during intralipid infusion. American Journal of Clinical Nutrition 32, 2416-2422.

Ota, D. M., Copeland, S. M., Corniere, J. N., Richie, E. K., Jacobson, K. \& Dudrick, S. J. (1978). The effects of a $70 \%$ soybean emulsion on lymphocyte transformation. Journal of Parenteral and Enteral Nutrition 2 , $112-115$.

Ota, D. M., Jessup, J. M., Babcock, G. F., Kirschbaum, L. \& Mountain, C. F. (1985). Immune function during intravenous administration of a soybean emulsion. Journal of Parenteral and Enteral Nutrition 9 , 23-27. 
Palmblad, J., Brostrom, O., Lahnborg, G., Uden, A. M. \& Venizelos, N. (1982). Neutrophil function during total parenteral nutrition and intralipid infusions. American Journal of Clinical Nutrition 35, 1430-1436.

Press, M., Kikuchi, H., Shimoyama, T. \& Thompson, G. R. (1974). Diagnosis of essential fatty acid deficiency in man. British Medical Journal 2, 247-249.

Rice, C., Hudig, D., Newton, R. S. \& Mendelsohn, J. (1981). Effect of unsaturated fatty acids on human lymphocytes: disparate influences of oleic acid and linoleic acids on natural cytotoxicity. Clinical Immunology and Immunopathology 20, 389-401.

Sedman, P. C., Ramsden, C. W., Brennan, T. G. \& Guillou, P. J. (1990). Pharmacological concentrations of lipid emulsions inhibit interleukin-2-dependent lymphocyte responses in vitro. Journal of Parenteral and Enteral Nutrition 14, 12-17.

Sedman, P. C., Somers, S. S., Ramsden, C. W., Brennan, T. G. \& Guillou, P. J. (1991). Effects of different lipid emulsions on lymphocyte function during total parenteral nutrition. British Journal of Surgery 78, 1396-1399.

Shamberger, R. C., Brennan, M. F. \& Goodman, J. T. (1982). A prospective randomised study of adjuvant parenteral nutrition in the treatment of sarcomas: results of metabolic and survival studies. Surgery 96, 1-6.

Stubbs, C. D., Tsang, W., Belin, J., Smith, A. D. \& Johnson, S. M. (1980). Incubation of exogenous fatty acids with lymphocytes. Changes in fatty acid composition and effects on the rotational relaxation time of 1,6-diphenyl-1,3,5-hexatriene. Biochemistry 19, 2756-2761.

Tebbey, P. W. \& Buttke, T. M. (1990). Molecular basis for the immunosuppressive action of stearic acid on T cells. Immunology 70, 379-384.

Tweedle, D. E. F. (1978). The use of fat emulsions in parenteral nutrition. In Advances in Parenteral Nutrition [I. D. A. Johnston, editor]. Lancaster: MTP Press Ltd. 